University of Wollongong

Research Online

Faculty of Engineering and Information

Faculty of Engineering and Information

Sciences - Papers: Part A

Sciences

$1-1-2014$

Discussion: prediction of long-term urban stormwater loads at single sites

Daniel May

University of Wollongong, dbm05@uow.edu.au

Muttucumaru Sivakumar

University of Wollongong, siva@uow.edu.au

Fahimeh Sarmadi

Imam Khomeini International University

Mohammad Azmi

Monash University

Mana Gharun

University of Sydney

Follow this and additional works at: https://ro.uow.edu.au/eispapers

Part of the Engineering Commons, and the Science and Technology Studies Commons

Research Online is the open access institutional repository for the University of Wollongong. For further information contact the UOW Library: research-pubs@uow.edu.au 


\title{
Discussion: prediction of long-term urban stormwater loads at single sites
}

\author{
Abstract \\ Contribution by Fahimeh Sarmadi, Mohammad Azmi and Mana Gharun May and Sivakumar (2013) have \\ predicted the long-term urban stormwater loads at single sites, using five different measures of central \\ tendency. They have stated that their motivation in using measures of central tendency is one in which, in \\ contrast to regression and process-based methods, there is no need for correlating urban stormwater \\ loads with other variables (e.g. discharge), as well as less complexity. Overall, the flow-weighted mean \\ concentration has produced the most accurate predictions of long-term loads.

\section{Disciplines} \\ Engineering | Science and Technology Studies \\ Publication Details \\ May, D., Sivakumar, M., Sarmadi, F., Azmi, M. \& Gharun, M. (2014). Discussion: prediction of long-term \\ urban stormwater loads at single sites. Proceedings of the Institution of Civil Engineers: Water \\ Management, 167 (8), 482-484.
}




\section{Discussion: Prediction of long-term urban stormwater loads at single sites}

Daniel May BE, PhD

Sustainable Water and Energy Research Group, School of Civil, Mining and Environmental Engineering, University of Wollongong, Wollongong, Australia

Muttucumaru Sivakumar ME, PhD, MIEAust, CPEng Associate Professor, Sustainable Water and Energy Research Group, School of Civil, Mining and Environmental Engineering, University of Wollongong, Wollongong, Australia

\section{Contribution by Fahimeh Sarmadi, Mohammad Azmi and Mana Gharun}

May and Sivakumar (2013) have predicted the long-term urban stormwater loads at single sites, using five different measures of central tendency. They have stated that their motivation in using measures of central tendency is one in which, in contrast to regression and process-based methods, there is no need for correlating urban stormwater loads with other variables (e.g. discharge), as well as less complexity. Overall, the flow-weighted mean concentration has produced the most accurate predictions of long-term loads. However, the following points are worth discussing:

The definite superiority of the flow-weighted mean method obviously shows that there is sufficient correlation between loads and runoff, and therefore it suggests that regression methods could derive more accurate results. Interestingly, the authors claim in a different study (May and Sivakumar, 2009a) that the comparison between different statistical models shows that regression models are more applicable than simple estimates of mean concentration in urban areas. Therefore, here, May and Sivakumar should compare different mean functions at least with a simple regression model to support their new claim.

From an environmental point of view, evaluating the trends, extreme events, dispersion tendency characteristics and bias are usually more effective than using a single value (e.g. long-term mean) for analysing/mitigating natural or human-induced side effects. For instance, Colin (1995) reports that urban stormwater loads have bias, which means the mode and arithmetic mean of samples are not related to the same load value; hence, pollution management for an area can be adjusted depending on the side of bias. In a different example, Vogel et al. (2005) state that the natural characteristics of long-term stormwater loads, at least in their case studies, are more sophisticated than using simple statistical methods. Therefore, Vogel et al. (2005) introduce a multivariate probability distribution function to model loadconcentration-discharge relationships in order to predict short and long-term stormwater loads. Furthermore, they derive benefit
Fahimeh Sarmadi MsC

Water Engineering Department, Imam Khomeini International University, Qazvin, Iran

Mohammad Azmi

PhD Candidate, Civil Engineering Department, Faculty of Engineering, Monash University, Melbourne, Australia

Mana Gharun

PhD Candidate, Faculty of Agriculture and Environment, University of Sydney, Sydney, Australia from using L-moment statistical analysis to discover the complexities of this phenomenon.

Overall, the current study presents valuable points; however, the title and concepts should clarify that the aim of the research is simply to compare the performance of different measures of central tendency in deriving site mean concentration, and not to predict long-term urban stormwater loads.

\section{Authors' reply}

The goal of the study by May and Sivakumar (2013) was to derive estimates of long-term load at a given catchment using a variety of estimates of site mean concentration (SMC) based on different measures of central tendency. The methodology was chosen for its inherent simplicity, making it readily applicable at sites with limited sampling.

Regression and/or process-based models are not solely limited by the absence of strong correlations between concentration and explanatory variables such as discharge, but also by a sparsity of available stormwater quality data, due to economic factors preventing the sampling of multiple storm events at a single catchment (Brezonik and Stadelmann, 2002; Driver and Tasker, 1990; Mourad et al., 2005a, 2006; Sliva and Williams, 2001). This sparsity of data makes it difficult to isolate typical trends accurately, supporting the use of simpler models such as SMC estimates. Furthermore, process-based models such as build-up wash-off models typically forecast the known relationship between stormwater load and runoff volume. This limits their ability to extract the more important unknown relationship between stormwater concentration and various environmental factors. In theory, regression models directly predicting stormwater concentration could overcome this limitation; however, previous studies conducted by May and Sivakumar (2009a, 2009b) do not necessarily support this hypothesis.

May and Sivakumar (2009a) found that long-term nutrient loads calculated using an estimate of SMC were more accurate than long-term loads calculated using multilinear regression models. 
Regression models were only superior to estimates of mean concentration from homogeneous land use categories and single metropolitan areas. Comparable results were found in another study conducted by May and Sivakumar (2009b), which sought to compare different methods aimed at predicting long-term heavy metal loads. Once again, long-term loads predicted using SMC estimates were more accurate than those derived from multilinear regression models, which were only superior to estimates of mean concentration from homogeneous land use categories and single metropolitan areas. The success of the flow-weighted mean concentration at predicting long-term loads does not necessarily imply that regression models will be superior to methods based on SMC. Even the simplest regression models have an additional calibration coefficient to SMC estimates, implying they will not perform well at sites with limited sampling, potentially overfitting on smaller datasets. This is supported by Mourad et al. (2005a, 2005b, 2005c) and Obropta and Kardos (2007) who suggested that SMCs required fewer data than regression or build-up washoff models. Furthermore, the study by May and Sivakumar (2013) found that the flow-weighted mean began performing less accurately on smaller datasets, potentially due to outlying storm event(s), which dominated the calibration of SMC. It is anticipated that this phenomenon would be even more pronounced on very small datasets, particularly if a regression approach with additional coefficients was adopted. Even though the flowweighted mean generally outperformed the other measures of central tendency, the lack of significant correlations between event mean concentration and runoff volume suggests the flowweighted mean is not as advantageous as it otherwise would be In particular, only two of the 17 catchments analysed had correlations significant at the $95 \%$ level. This finding is supported by additional studies conducted by Athayde et al. (1983) and Huber (1992), which observed a lack of correlation between concentration and a variety of explanatory variables such as runoff volume. These observations suggest that regression models are not likely to be significantly more accurate than the simpler SMC estimates. It is also anticipated that annual pollutant loads would be difficult to generate using regression models, as opposed to simple SMC estimates. Once the SMC has been determined at a given catchment, it may be multiplied by the annual runoff volume to provide a readily attainable estimate of annual load.

There are a number of methods for determining the annual runoff volume, the simplest derived by multiplying the mean annual rainfall estimate by the runoff coefficient at a given catchment. In contrast, regression techniques require a 'typical' set of storm runoff volumes over a period of a year. While these data may be available at certain catchments, the 'typical' set chosen may not be representative of the catchment. As a result, significant variability in annual load estimates may be observed depending on the year chosen for analysis, making it difficult to isolate a single estimate of annual runoff load. Furthermore, confidence intervals may not be easily calculated using a regression modelling approach, as opposed to simpler methods based on SMC.
Even the flow-weighted mean allows the calculation of an associated standard deviation, although the chosen method may not be universal (Mourad et al., 2005a). The associated confidence interval subsequently allows the modeller to determine the reliability of the estimate of annual load from the catchment of interest, and the likelihood of exceeding a specified load input into a receiving water.

Unlike the article by May and Sivakumar (2013), the methods defined by Colin (1995) and Vogel et al. (2005) do not seek to predict long-term urban stormwater loads. Instead, they aim to forecast pollutant loads exported within large river systems, much larger than the urban catchments analysed in the study by May and Sivakumar (2013). The methods defined by Colin (1995) and Vogel et al. (2005) also typically rely on continuous sampling data, containing both dry weather flow and runoff from storm events, whereas the method defined by May and Sivakumar (2013) focuses solely on discrete runoff events monitored sporadically. These inherent differences in the type of data suggest different methodologies would be applicable in each context. In particular, a sparsity of stormwater data available at most urban catchments implies the need for simpler models with fewer calibration parameters, such as SMC estimates.

Overall, the objective of the study by May and Sivakumar (2013) was to forecast long-term urban stormwater loads using different estimates of SMC. This is of particular importance when determining long-term phosphorus inputs into receiving water prone to eutrophication, with response times in the order of several years (Huber, 1992). However, SMCs may be used for other purposes, deemed outside the scope of the study. For example, they may be combined with discrete runoff volumes to generate stormwater loads from extreme events, or to generate stormwater load distributions at a given catchment. Although more complex methods are available, it is the inherent simplicity of the SMC method that makes it readily applicable to most catchments, particularly those with limited sampling data.

\section{REFERENCES}

Athayde DN, Shelley PE, Driscoll ED, Gaboury D and Boyd G (1983) Results of the Nationwide Urban Runoff Program. US Environmental Protection Agency, Washington, DC, USA, vol. 1, PB84-1855345.

Brezonik PL and Stadelmann TH (2002) Analysis and predictive models of stormwater runoff volumes, loads and pollutant concentrations from watersheds in the Twin Cities metropolitan area, Minnesota, USA. Water Research 36(7): $1743-1757$.

Colin TA (1995) Recent advances in statistical methods for the estimation of sediment and nutrient transport in rivers. US Report to the International Union of Geodesy and Geophysics, 1991-1994. Reviews of Geophysics 33(52): 1117-1123.

Driver N and Tasker G (1990) United States Geological Survey. Techniques for Estimation of Storm-Runoff Loads, Volumes 
and Selected Constituent Concentrations in Urban Watersheds in the United States. United States Government Printing Office, Washington, DC, USA, Water Supply Paper 2363.

Huber WC (1992) Prediction of urban nonpoint source water quality: methods and models. Proceedings of International Symposium on Urban Stormwater Management, Institution of Engineers, Sydney, Australia, pp. 1-16.

May D and Sivakumar M (2009a) Prediction of nutrient concentrations in urban storm water. Journal of Environmental Engineering (ASCE) 135(8): 586-594.

May D and Sivakumar M (2009b) Prediction of heavy metal concentrations in urban storm water. Water and Environment Journal 23(4): 247-254.

May D and Sivakumar M (2013) Prediction of long-term urban stormwater loads at single sites. Water Management 166(2): 81-92.

Mourad M, Bertrand-Krajewski JL and Chebbo G (2005a) Sensitivity to experimental data of pollutant site mean concentration in stormwater runoff. Water Science and Technology 51(2): 155-162.

Mourad M, Bertrand-Krajewski JL and Chebbo G (2005b)
Stormwater quality models: sensitivity to calibration data. Water Science and Technology 52(5): 61-68.

Mourad M, Bertrand-Krajewski JL and Chebbo G (2005c) Calibration and validation of multiple regression models for stormwater quality predictions: data partitioning, effect of dataset size and characteristics. Water Science and Technology 52(3): 45-52.

Mourad M, Bertrand-Krajewski JL and Chebbo G (2006) Design of a retention tank: comparison of stormwater quality models with various levels of complexity. Water Science and Technology 54(6-7): 231-238.

Obropta CC and Kardos JS (2007) Review of urban stormwater quality models: deterministic, stochastic, and hybrid approaches. Journal of the American Water Resources Association 43(6): 1508-1523.

Sliva L and Williams DD (2001) Buffer zone versus whole catchment approaches to studying land use impact on river water quality. Water Research 35(14): 3462-3472.

Vogel RM, Rudolph BE and Hooper RP (2005) Probabilistic behaviour of water-quality loads. Journal of Environmental Engineering (ASCE) 131(7): 1081-1089. 